\title{
Evaluación de un bi-epítopo de gliceraldehídmo 3-fosfato deshidrogenasa y de Desorganización Muscular en la protección de ovinos contra Haemonchus contortus
}

\author{
Evaluation of a bi-epitope of glyceraldehyde 3-phosphate dehydrogenase and \\ Disorganized Muscle in the protection of sheep against Haemonchus contortus
}

\author{
Arnielis Díaz', Yipsi García ${ }^{1,4}$, Yuliet Sánchez ${ }^{1}$, Andreia Buzatti², Yanexis Rodríguez ${ }^{3}$, \\ Maritza Infante $^{3}$, Ubaldo Torres ${ }^{3}$, Amilcar Arenal ${ }^{1}$, Marcelo Molento ${ }^{2}$
}

\section{RESUMEN}

El objetivo del estudio fue evaluar la capacidad de protección en ovinos contra Haemonchus contortus de un péptido que mimifica epítopos de gliceraldehído 3-fosfato deshidrogenasa (GAPDH) y Desorganización Muscular (DIM-1) de H. contortus. Se hizo una búsqueda en una biblioteca de fagos (7 aa-GGG) con el empleo de un anticuerpo policlonal (IgG) anti-H. contortus. Después de tres rondas de selección se secuenciaron 120 clones y se hizo el análisis de secuencia de cada clon. Se seleccionaron clones cuya secuencia de péptidos fue similar a la proteína de DIM-1 y a la GAPDH, y se sintetizó un péptido con estos dos epítopos. Los animales se inmunizaron los días $0,7,14$ y 28 . Una semana después de la última vacunación todos los animales se desafiaron con 1250 L3 de $H$. contortus. La media de huevos por gramo de heces (hpg) y la carga parasitaria en el abomaso del grupo vacunado a los 70 días de la infestación se redujeron en 57.9 y $52.7 \%$, respectivamente. Los niveles de IgG en suero fueron significativamente más altos en el grupo vacunado; además aumentó el número de eosinófilos y subió el valor de la hemoglobina.

Palabras clave: DIM-1; GAPDH; biblioteca de fagos; péptidos; multiepítopos

\section{Abstract}

The aim of this study was to evaluate the protection capacity in sheep against Haemonchus contortus using a peptide that mimics epitopes of glyceraldehyde 3phosphate dehydrogenase (GAPDH) and the Disorganized Muscle (DIM-1) of $H$.

\footnotetext{
${ }^{1}$ Laboratorio de Bioquímica, Departamento de Morfofisiología, Universidad de Camagüey, Cuba

${ }^{2}$ Laboratorio de Enfermedades Parasitarias, Universidad Federal de Paraná, Curitiba, Brasil

${ }^{3}$ Departamento de Bioquímica, Universidad de Ciencias Médicas de Camagüey Carlos J. Finlay, Camagüey, Cuba

${ }^{4}$ E-mail: yipsi.garcia@reduc.edu.cu
}

Recibido: 14 de diciembre de 2017

Aceptado para publicación: 2 de septiembre de 2018 
contortus. A genotypic phage library (7 aa-GGG) was evaluated with the use of a polyclonal anti-H. contortus antibody IgG. After three rounds of selection, 120 clones were sequenced and the amino acid sequences were aligned. Peptide sequences similar to DIM-1 and GAPDH were selected, and a peptide with these two epitopes was synthesized. A group of four rams with the bi-epitope and another similar group with the adjuvant were immunized on days $0,7,14$ and 28 . One week after the last vaccination all the animals were challenged with $1250 \mathrm{~L} 3$ larvae of $H$. contortus. The average number of eggs per gram of feces (hpg) and the parasitic load in the abomasum of the group vaccinated 70 days after the infestation were reduced by 57.9 and $52.7 \%$, respectively. Serum IgG levels were significantly higher in the vaccinated group; it also increased the number of eosinophils and increased the hemoglobin.

Keywords: DIM-1; GAPDH; phage library; peptides; multiepitopes

\section{INTRODUCCIÓN}

Los métodos actuales para el control de nematodos gastrointestinales se basan principalmente en el empleo de antihelmínticos químicos, cuyo uso indiscriminado durante largo tiempo provoca el desarrollo de la resistencia de los parásitos al tratamiento. Además, se describe la contaminación del medio ambiente y la presencia de residuos tóxicos en la cadena alimentaria que llega hasta el hombre (Wardhaugh, 2005).

Una alternativa de control de los nematodos gastrointestinales puede ser la inmunización con proteínas nativas. La H11 de H. contortus es una glicoproteína eficaz como inmunógeno (Tavernor et al., 1992). La vacunación con extractos de proteínas de excreción/secreción de $H$. contortus también es efectiva en la protección de la infestación parasitaria (Lacroux et al., 2006). La purificación de proteínas de excreción/secreción como la HC58 (cisteína proteasa y galectina) reducen parcialmente la infección que produce H. contortus (Muleke et al., 2007; Yanming et al., 2007). No obstante, la obtención de pequeñas cantidades de estas proteínas por vía natural es una limitante para convertirlas en un producto comercial, de allí que la búsqueda de otras alternativas como la tecnología del ADN recombinante se convierte en una necesidad.
El uso de proteínas recombinantes presenta limitaciones para el control de los parásitos, ya que no contienen las modificaciones pos-traduccionales apropiadas para retener la capacidad protectora de las moléculas naturales; por ejemplo, carecen o forman puentes disulfuros erróneos, otro o ningún patrón de glicosilación y diferente plegamiento en el espacio (Newton y Meeusen, 2003).

En el trabajo de Yan et al. (2010) sobre inmunoproteómica de hembras y machos adultos de $H$. contortus se describen 23 proteínas del metabolismo o estructurales, con alta identidad de proteínas en nematodos, entre ellas, la enzima GAPDH y DIM-1.

DIM-1 pertenece a la superfamilia de las inmunoglobulinas. Es una proteína estructuralmente localizada en la región de la membrana celular del músculo alrededor de los cuerpos densos y se ancla en los filamentos de actina. Esta puede desempeñar un papel en la estabilización de los componentes finos y filamentosos del sarcómero (Rogalski et al., 2003). DIM-1 es altamente conservada a nivel de aminoácidos con su homólogo en nematodos como Caenorhabditis briggsae (99\%), Brugia malayi $(77 \%) \mathrm{y}$ Ascaris suum (77\%). El antígeno AS37 de A. suum, con alta identidad con DIM-1, se reconoce por sueros de ovejos infestados con L3 (Tsuji et al., 2002). Un estudio preliminar sugiere que la vacuna de ADN DIM-1 indu- 
ce respuesta inmune parcial en cabras contra el $H$. contortus (Yan et al., 2013).

Gliceraldehído 3-fosfato deshidrogenasa muestra un potencial de protección como inmunógeno frente a diferentes helmintos. En Schistosoma mansoni, GAPDH produce respuestas significativas de células B y células $\mathrm{T}$, e induce inmunidad protectora (Erttmann et al., 2005). La aplicación del gen de GAPDH como candidato vacunal en ovejas induce protección parcial frente a $H$. contortus (Han et al., 2012).

Los recientes avances en la tecnología de bibliotecas de fago llevan esta metodología a convertirse en una herramienta valiosa para la investigación (Han et al., 2012). Por ejemplo, en el empleo para mimificar epítopos de parásitos como Fasciola hepatica (VillaMancera et al., 2008), Rhipicephalus (Boophilus) microplus (Prudencio et al., 2010), Taenia solium (Assana et al., 2010), Schistosoma japonicum (Wu et al., 2006) y Plasmodium falciparum (Ambroggio et al., 2013). En helmintos se recomienda el empleo de vacunas multiepítopos debido a la complejidad de los mecanismos de infestación (Gnanasekar et al., 2004; Wu et al., 2006); sin embargo, no se ha descrito su uso en $H$. contortus. El objetivo del estudio fue evaluar la capacidad de protección en ovinos contra Haemonchus contortus de un péptido que mimifica epítopos de gliceraldehímo 3fosfato deshidrogenasa (GAPDH) y Desorganización Muscular (DIM-1) de Haemonchus contortus.

\section{Materiales y Métodos}

Los experimentos se realizaron bajo el protocolo del Comité de Ética de la Facultad de Ciencias Agropecuarias de la Universidad de Camagüey (01/2013).

\section{Material Biológico}

Se extrajeron muestras de sangre $(10$ $\mathrm{ml})$ de la vena yugular de 48 carneros adul- tos que se encontraban bajo pastoreo. El suero se obtuvo por centrifugación a $1500 \mathrm{~g}$ durante 10 min y se almacenó a $-20^{\circ} \mathrm{C}$ hasta su análisis por ELISA.

Cinco gramos de $H$. contortus adultos, obtenidos de un animal sacrificado en un matadero y que tenía una alta carga parasitaria, fueron macerados en nitrógeno líquido. Las proteínas se extrajeron con Tritón X-100 al 1\% [volumen/volumen] ([v/v]) en TFS 1X (tampón fosfato salino) $\left(\mathrm{NaCl} 136.8 \mathrm{mmol} \mathrm{L}^{-1}\right.$; $\mathrm{KCl} 2.7 \mathrm{mmol} \mathrm{L}^{-1} ; \mathrm{Na}_{2} \mathrm{HPO}_{4} 10.2 \mathrm{mmol} \mathrm{L}^{-1}$; $\mathrm{KH}_{2} \mathrm{PO}_{4} 1.8 \mathrm{mmol} \mathrm{L}^{-1}, \mathrm{pH}$ 7.1). Las proteínas se cuantificaron por el método de Bradford (1976).

\section{ELISA}

Las placas de ELISA se recubrieron con $5 \mu \mathrm{g} / \mathrm{ml}$ de la proteína de $H$. contortus (o un péptido sintético en el caso indicado), diluido en tampón carbonato $\left(34.9 \mathrm{mmol} \mathrm{L}^{-1}\right.$ $\mathrm{NaHCO}_{3}, 15$ mmol L-1 $\mathrm{Na}_{2} \mathrm{CO}_{3} ; \mathrm{pH}$ 9.6). Se siguió la metodología descrita por Díaz et al. (2015). Las placas se leyeron a $450 \mathrm{~nm}$ en el lector de ELISA de microplacas Biotek (Gen5). Controles positivos y negativos estándar se incluyeron en cada placa. Los controles positivos fueron sueros de los animales que tenían altos títulos, mientras que los controles negativos fueron sueros de animales que la lectura estuvo por debajo de la línea de corte. Los sueros negativos empleados para determinar la línea de corte fueron obtenidos de 18 animales estabulados que nunca estuvieron contacto con $H$. contortus. La línea de corte fue determinada por análisis ROC. La sensibilidad del test fue de 100\% y la especificidad de $94.44 \%$.

\section{Selección en Biblioteca de Fago}

La búsqueda se hizo utilizando una biblioteca de fagos de 7-mer (7 aa-GGG) (Cat. E8100S, New England Biolabs). El protocolo de selección se realizó según las instrucciones del fabricante. Se realizaron tres rondas de selección por afinidad. El suero de un animal con altos títulos contra $H$. contortus se 
recubrió a una dilución de 1:100 en tampón carbonato. Las placas se incubaron a $4{ }^{\circ} \mathrm{C}$ durante la noche. Luego, las placas se bloquearon con tampón de bloqueo (TFST suplementado con 5\% de BSA) a temperatura ambiente durante $1 \mathrm{~h}$. Las placas se lavaron tres veces con TFST. Se tomó $10 \mu 1 \mathrm{del}$ bacteriófago $\left(10^{11}\right.$ unidades formadoras de placas [pfu]) con péptidos de 7-mer (7 aaGGG) diluidos en TFST y se añadió al suero negativo para evitar interacciones inespecíficas. Se colocó el contenido en el suero positivo. La placa se lavó con TFST; luego, los fagos absorbidos se eluyeron con glicina $0.1 \mathrm{~mol} \mathrm{~L}^{-1} \mathrm{pH} 2.5$ y se neutralizaron con Tris- $\mathrm{HCl} \mathrm{pH}$ 8. Los fagos eludidos se amplificaron mediante la infección de Escherichia coli y se purificaron por precipitación con $20 \%$ [peso/volumen] de PEG $8000 / \mathrm{NaCl} 2.5 \mathrm{~mol} \mathrm{~L}^{-1}$.

\section{Secuenciación}

El ADN de los clones de fagos positivos se extrajo con fenol cloroformo. Las muestras se secuenciaron por capilaridad (ABI3130, BigDie v. 3.1 y POP 7 polímero) (Life Technologies/Thermo Fisher Scientific, Myleus, Brasil). Después se realizó el análisis bioinformático del ADN secuenciado con el empleo del Vector NTI v. 7.0 (https:// www.thermofisher.com/us/en/home/lifescience/cloning/vector-nti-software.html).

\section{Vacunación}

Se utilizaron ocho carneros de 12-13 meses y con peso de $53 \pm 2 \mathrm{~kg}$. Los animales fueron estabulados y desparasitados $28 \mathrm{y}$ 14 días antes del inicio del experimento. El día 0 se inicia el experimento con la primera vacunación de los animales. Los ovinos se dividieron en dos grupos, uno $(n=4)$ fue vacunado con el bi-epítopo GAPDH y DIM-1 (Mlu2: GSGNAIMGGGSFLSDTRS, PM $1713.83 \mathrm{gmol}^{-1}, 78.2 \%$ pureza en Genescript, EEUU) a $2 \mu \mathrm{g} / \mathrm{kg}$ de peso del animal y adyuvante (Montanide 50VG-2). El grupo placebo $(\mathrm{n}=4)$ solo recibió el adyuvante. Se realizaron cuatro inmunizaciones en sema- nas consecutivas y los animales fueron infestados con 1250 larvas L3 de H. contortus el día 35 del experimento. Las larvas se obtuvieron por la técnica de Bearmann de un coprocultivo de huevos colectados a partir de la ovoposición de $H$. contortus adultos del animal sacrificado en matadero (MAFF, 1986).

Se colectaron muestras de sangre con EDTA los días 0, 35 y 70 del experimento. Se determinó el hematocrito por el método del micro-hematocrito y la hemoglobina por el método de la cianometahemoglobina, y se realizó el recuento diferencial de células. Las muestras de heces fueron colectadas directamente del recto los días $0,14,28,35,42$, $49,56,63$ y 70 y el número de huevos por gramo de heces (HPG) fue determinado a través del método de McMaster (Gordon and Whitlock, 1939). Todos los carneros de los dos grupos fueron sacrificados a los 70 días del experimento y se contaron los nemátodos adultos del abomaso.

\section{Análisis Estadístico}

Las comparaciones entre grupos se realizaron por la prueba no paramétrica de Mann Whitney. Se empleó el paquete estadístico SPSS para Windows 19.0 (SPSS Inc., Chicago, IL, EEUU).

\section{Resultados y Discusión}

\section{Evaluación de la Respuesta Natural}

La Figura 1 muestra la respuesta inmunológica (IgG) de los 48 animales que estaban en pastoreo contra proteínas del extracto de $H$. contortus. La respuesta natural en el $71 \%$ de los animales fue superior a 1.25 de densidad óptica. Todos los valores de absorbancia quedaron situados por encima de la línea de corte, lo que indica que todos han tenido contacto con el parásito y han generado anticuerpos contra esta infestación natural. Esto concuerda con la respuesta natural 


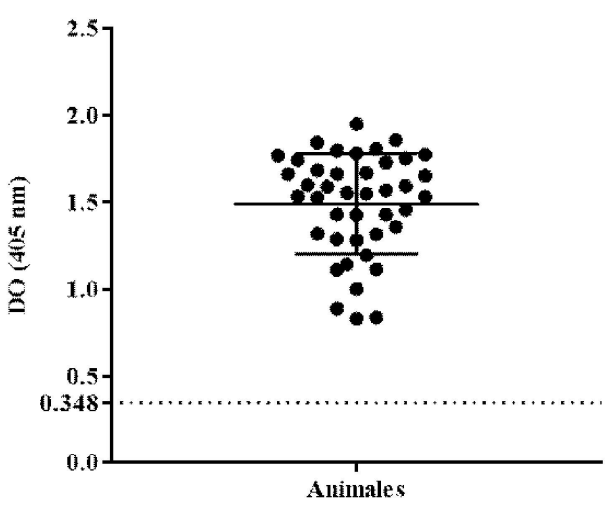

Figura 1. Detección de títulos de IgG antiHaemonchus contortus en sueros de 48 carneros adultos a través de ELISA con placas recubiertas con proteína de un extracto de $H$. contortus. La barra horizontal indica la media de los títulos. Las T muestran la desviación estándar. La línea discontinua representa el valor de la línea de corte (según análisis ROC)

de IgG e IgA que se describe en ovejas cuando se infestan al pastar (Terefe et al., 2007). La respuesta natural resulta independiente del nivel de infestación y protege a los animales de una reinfestación. Los niveles de título de IgG están relacionados con la protección del hospedero (LeJambre et al., 2008). Así mismo, se reporta que cruces genéticos de ovinos con mayor resistencia a $H$. contortus presentan mayores títulos de IgG (Shakya et al., 2011). En este estudio, todos los animales con altos títulos $(>1.25)$ presentaban bajo HPG (0-200 huevos), lo que confirma que esta respuesta los hace resistente al parásito.

\section{Selección en una Biblioteca de Fago}

De 120 clones aislados a partir del pesquisaje en una biblioteca de fago con un suero de altos títulos de anticuerpos que reconocen proteínas de $H$. contortus, solo nueve mostraron alta identidad con las 23 proteínas declaradas en la inmunoproteómica de adultos del parásito (Yan et al., 2010). La Figura 2 muestra el alineamiento de los clones $(46,103,32,90,69,66,60,111,117)$ con pro- teínas endógenas de $H$. contortus. El análisis de secuencia de los clones positivos reveló alta identidad entre ellos y con algunas de las proteínas que forman parte de la constitución de $H$. contortus. El clon 46 mostró identidad en cuatro (Gly, Ala, His, Asp) de sus siete aminoácidos con la proteína glutamato deshidrogenasa. De manera similar ocurrió con los clones 103, 32 y 90 que mostraron una secuencia consenso con alta identidad a la ATPasa de segregación de cromosoma, ya que cuatro aminoácidos (Glu, Ala, Leu, Thr) coinciden con los de esta proteína. Asimismo, el clon 69 también presentó identidad con un fragmento de la glutatión serín transferasa, con cuatro aminoacidos que coinciden y otros dos con estructuras químicamente parecidas (Asp-Glu e Ile-Val).

La secuencia peptídica correspondiente al clon 66 es la de mayor identidad (71.4\%) con un fragmento de gliceraldehído 3-fosfato deshidrogenasa, ya que cinco aminoácidos son iguales (Phe, Ser, Asp, Thr, Ser) y uno químicamente semejante (Leu-Val). Por su parte la secuencia peptídica correspondiente del clon 60 posee solo dos Phe iguales y tres aminoácidos químicamente parecidos (AspGlu, Phe-Tyr y Met-Leu). Por último, la secuencia correspondiente a los clones 111 y 117 presenta cuatro aminoácidos idénticos a un fragmento de DIM-1 (Gly, Gly, Asp, Ala), además de uno químicamente similar (Ser-Lys).

A pesar de secuenciar 120 clones no fue posible obtener secuencias consensos, posiblemente porque los sueros naturales reconocen varios epítopos (de diferentes proteínas) al mismo tiempo. Prudencio et al. (2010) trabajaron con una biblioteca de fago presentadora de péptidos, con un anticuerpo policlonal para garrapata, sin observar identidad después de secuenciar 36 clones. Así mismo, Villa-Mancera et al. (2008) identificaron siete secuencias no consenso con un antisuero para catepsina L1/L2 de Fasciola hepatica. Arnon et al. (2000), usando un anticuerpo monoclonal que protege frente a la infección de Schistosoma mansoni no detectaron secuencias consenso, por ejem- 

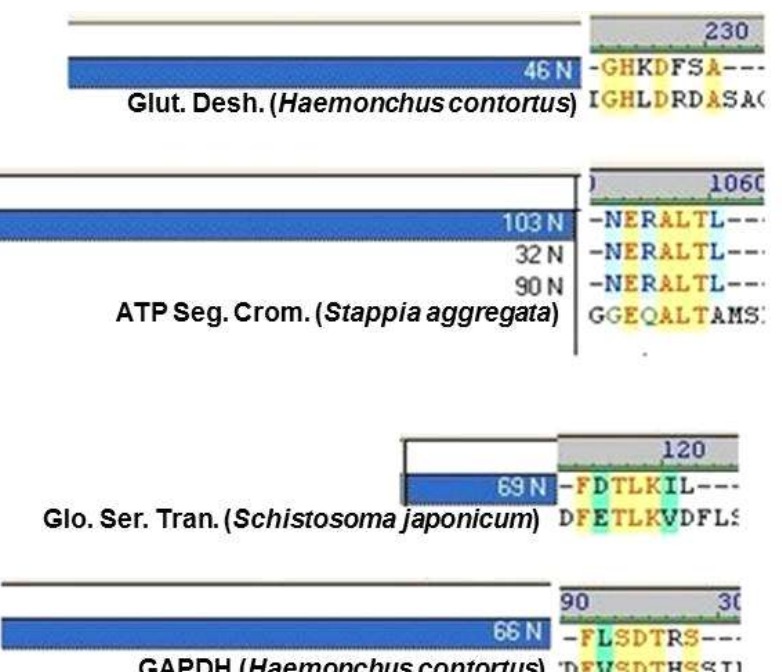

GAPDH (Haemonchus contortus) DFVSDTHSSI

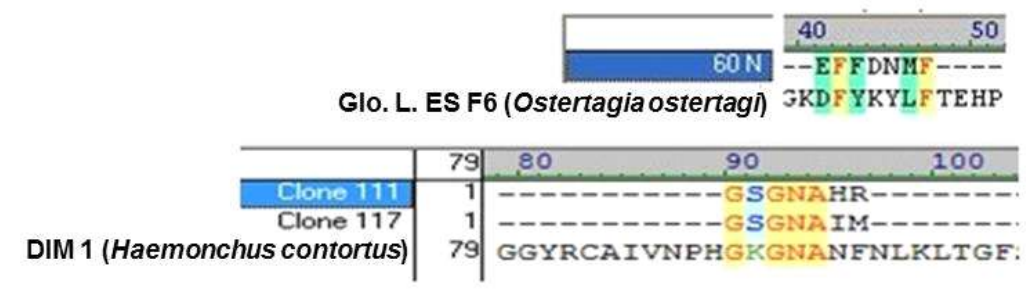

Figura 2. Mayor identidad de péptidos provenientes de los clones de la biblioteca de fagos alineados con diferentes proteínas registradas en bases de datos internacionales. Glut. Desh.: glutamato deshidrogenasa; ATP Seg. Crom.: ATPasa de segregación cromosomal; Glo. Ser. Tran.; globin serin transferasa; GAPDH; Glo. L. ES F6: globinlike de excresión/secresión proteína F6; DIM 1: proteína de Desorganización Muscular

plo, con Schistosoma japonicum (Tang et al., 2004; Liu et al., 2011), Entamoeba histolytica (Melzer et al., 2002) y Rhipicephalus microplus (Bishop-Hurley et al., 2010).

\section{Vacunación}

La Figura 3 muestra el conteo de huevos por gramo de heces (hpg) de los animales de cada grupo experimental. La ovoposición comenzó a los 63 días (28 días después de la infestación), lo que coincide con el periodo de prepatencia del $H$. contortus. El promedio de hpg el día 70 (día del sacrificio) fue $57.9 \%$ menor $(2130 \mathrm{hpg}$ ) en el grupo vacunado con el péptido Mlu2 que en el grupo control $(5060 \mathrm{hpg})(\mathrm{p}<0.05)$.
La carga de parásitos adultos en el abomaso de los animales del grupo vacunado fue $52.3 \%$ menor que en el grupo control $(\mathrm{p}<0.01)$. La proporción de hembras en el grupo vacunado $(55 / 104)$ fue similar al grupo control (116/218). Para Sargison et al. (2011), el nivel de infestación puede variar por la edad de los animales, la supresión inmune y las cepas de $H$. contortus presentes; así como el número de larvas que se usen para el desafío. Yan et al. (2013) reportaron en sus estudios 8000 hpg y 524 nemátodos adultos, mientras que Zhao et al. (2012) hallaron 13000 hpg y 742 nematodos adultos.

El gliceraldehido 3-fosfato deshidrogenasa es uno de los péptidos de más empleo en ensayos de vacunación para proteger con- 


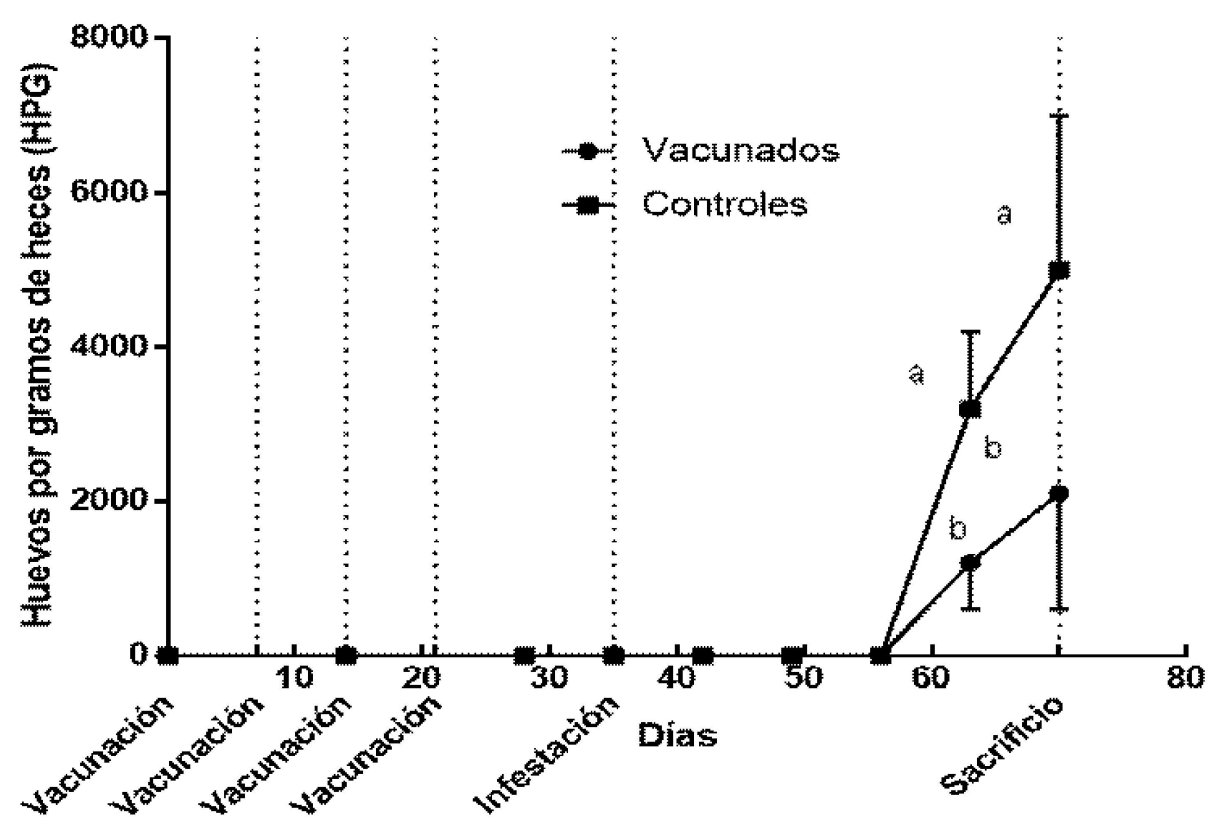

Figura 3. Dinámica de huevos por gramos de heces (media \pm d.e.) en carneros vacunados con el bipéptido Mlu2 (GSGNAIMGGGSFLSDTRS). El día 0 corresponde al primer día de la vacunación. El control fue un placebo vacunado solamente con el adyuvante. Los dos grupos fueron infestados con $1250 \mathrm{~L} 3 \mathrm{de} \mathrm{H}$. contortus. Las líneas discontinuas indican los días de vacunación, el día de la infestación y el momento del sacrifico. Las T muestran la desviación estándar. Letras diferentes indican diferencias significativas $(\mathrm{p}<0.05)$.

tra parásitos gastrointestinales. En Schistosoma mansoni se evaluaron seis péptidos deducidos de la secuencia primaria de GAPDH, y con la combinación de cuatro de ellos redujeron significativamente el hpg y la carga parasitaria. La vacunación de cabras con ADN de GAPDH de $H$. contortus redujo la producción de huevos $(34.9 \%)$ y la carga parasitaria en el abomaso (37.7\%) (Yan et al., 2013). Aunque los resultados del presente estudio son superiores, se reafirma el hecho de usar GAPDH como candidato vacunal contra $H$. contortus, que en este caso es un péptido que mimifica la respuesta inmunológica.

La proteína de Desorganización Muscular 1 (DIM-1) es un nuevo candidato como antígeno para proteger frente a diversos parásitos. La vacunación en cabras con el gen de DIM-1 redujo la producción de huevos y carga parasitaria en el abomaso en 45.7 y $51.1 \%$, respectivamente (Yan et al., 2013). Así mismo, la inyección de BS-Ag3 (idéntica a DIM-1) de Baylisascaris schroederi (ascariasis de panda) redujo significativamente en $62.9 \%$ la carga de parásitos (Wang et al., 2008). Los resultados del presente estudio confirman los hallazgos de usar esta proteína como candidato vacunal. Aunque el mecanismo de protección con el uso de DIM-1 y GAPDH como antígenos es aún desconocido, se discute que puede estar dada por el incremento de anticuerpos y eosinófilos.

El número de eosinófilos fue más alto en el grupo vacunado que en el grupo control $(p<0.01)$. Así mismo, el valor de hemoglobina fue menor en el grupo control a partir del día 56 , siendo $20 \%$ menor el día del sacrificio (Cuadro 1). El aumento de eosinófilos en sangre periférica es considerado como un mar- 


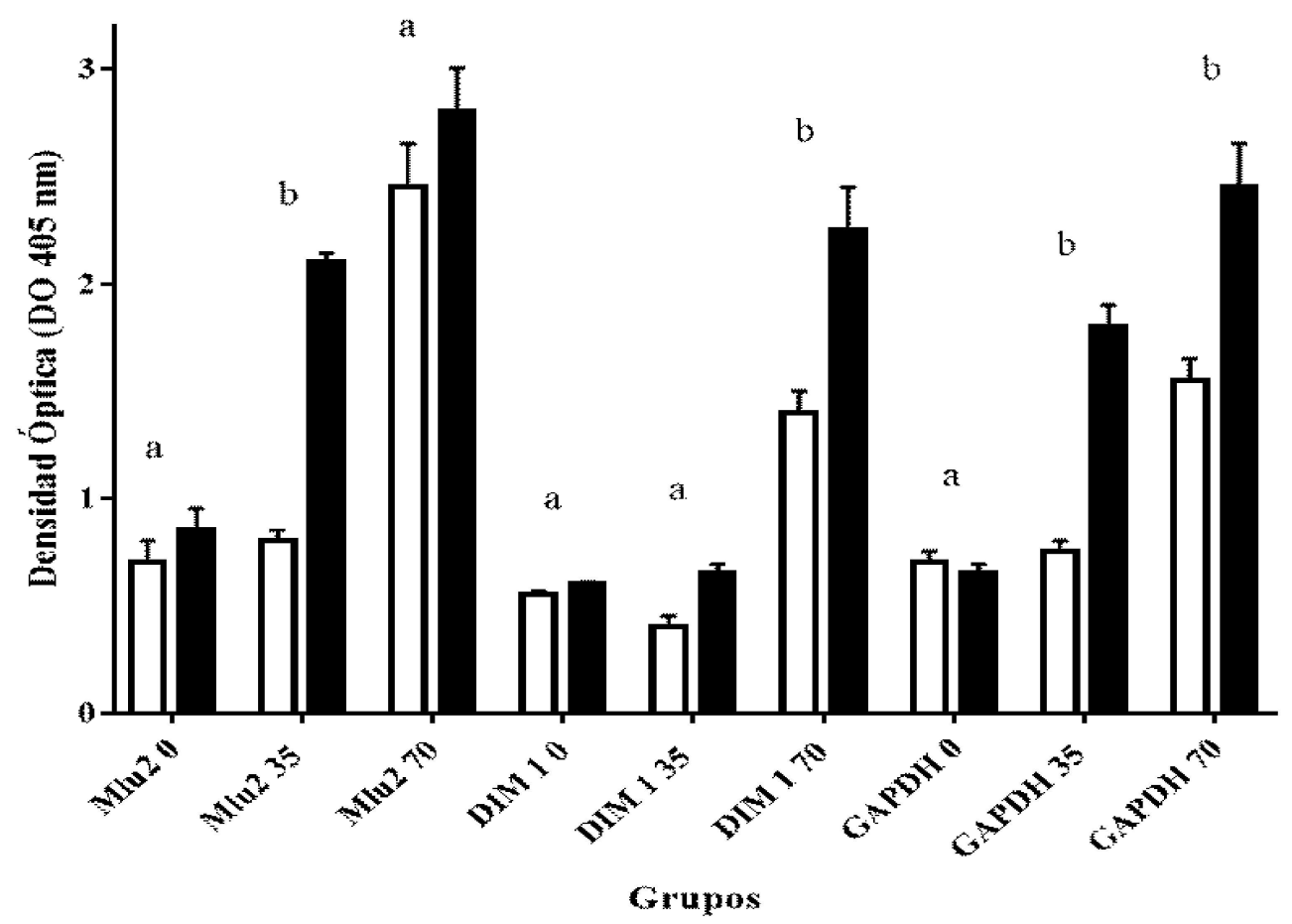

Figura 4. Títulos de IgG que reconocen los péptidos Mlu2 (GSGNAIMGGGSFLSDTRS), DIM1 (GSGNAIM), GAPDH (FLSDTRS). 0: primera vacunación. 35: día de la infestación; 70: día del sacrificio. Las T muestran la desviación estándar. Letras diferentes indican diferencias significativas $(\mathrm{p}<0.05)$

cador potencial del hospedero para la resistencia contra H. contortus (Douch et al., 1996). Los eosinófilos pueden dañar y probablemente matar las larvas del parásito en el tracto gastrointestinal (Balic et al., 2006). En un estudio in vitro, eosinófilos extraídos de lavados mamarios de ovejas infestadas repetidamente, inhibieron la movilidad y mataron las L3 de H. contortus en presencia de anticuerpo de superficie anti-L3 (Rainbird et al., 1998).

Los niveles en sangre de IgG que reconocen el epítopo fueron máximos en el día del sacrificio. El mismo comportamiento se observó para ambos péptidos independientes; aunque en el caso de GAPDH hubo una respuesta más rápida, comparado con DIM-1 en el momento de la infestación y en el sacrificio (Figura 4).
Altos títulos de IgG específicos se relacionan con la defensa del huésped contra el desafío de $H$. contortus. Por ejemplo, la inmunización con una fracción de la proteasa catepsina B provocó altos títulos de IgG e indujo una reducción del $47 \%$ en la carga de parásitos y del $77 \%$ de la producción de huevos (Knox y Smith, 2001). Además, el aumento de los niveles séricos de IgG1 e IgG2 se asociaron con el recuento fecal de huevos disminuido (Schallig et al., 1995). De manera similar, en ovejas resistentes se encontraron antígenos para producir mayores niveles de IgG1 e IgE (Gill et al., 1993). Los resultados aquí descritos fueron consistentes con informes anteriores, porque confirman que el mecanismo de protección puede estar mediado por anticuerpos y la participación de los eosinófilos. 
Cuadro 1. Recuento de eosinófilos y niveles de hemoglobina y hematocrito en cuatro corderos vacunados contra Haemonchus contortus y cuatro corderos control no vacunados

\begin{tabular}{lccc}
\hline & $\begin{array}{c}\text { Tiempo } \\
\text { (días) }\end{array}$ & Control & $\begin{array}{c}\text { Vacunados } \\
(\text { Mlu2 })^{1}\end{array}$ \\
\hline Eosinófilos $\left(1 \times 10^{9}\right) \mathrm{mL}^{-1}$ & 0 & $0 \pm 0^{\mathrm{a}}$ & $0 \pm 0^{\mathrm{a}}$ \\
& 28 & $0.01 \pm 0^{\mathrm{b}}$ & $0.07 \pm 0.03^{\mathrm{a}}$ \\
& 56 & $0.06 \pm 0.02^{\mathrm{b}}$ & $0.15 \pm 0.02^{\mathrm{a}}$ \\
Hemoglobina $\left(\mathrm{g} \mathrm{L}^{-1}\right)$ & 70 & $0.08 \pm 0.04^{\mathrm{b}}$ & $0.20 \pm 0.07^{\mathrm{a}}$ \\
& 0 & $102.2 \pm 5.8^{\mathrm{a}}$ & $100.6 \pm 14.3^{\mathrm{a}}$ \\
Hematocrito $(\%)$ & 28 & $91.4 \pm 6.8^{\mathrm{a}}$ & $91.0 \pm 7.4^{\mathrm{a}}$ \\
& 56 & $74.2 \pm 5.3^{\mathrm{b}}$ & $87.2 \pm 3.8^{\mathrm{a}}$ \\
& 70 & $62.6 \pm 7.0^{\mathrm{b}}$ & $77.4 \pm 6.9^{\mathrm{a}}$ \\
& 0 & $33.8 \pm 2.2^{\mathrm{b}}$ & $33.1 \pm 2.9^{\mathrm{a}}$ \\
& 28 & $31.1 \pm 3.2^{\mathrm{b}}$ & $32.0 \pm 1.8^{\mathrm{a}}$ \\
& 56 & $27.3 \pm 4.0^{\mathrm{a}}$ & $31.5 \pm 7.9^{\mathrm{a}}$ \\
& 70 & $20.7 \pm 3.5^{\mathrm{b}}$ & $25.5 \pm 2.4^{\mathrm{a}}$ \\
\hline
\end{tabular}

${ }^{1}$ Mlu2 bipéptido (GSGNAIMGGGSFLSDTRS, PM 1713.83 gmol-1, 78.2\% pureza en Genescript, EEUU ) que contiene los epítopos de DIM-1 y GAPDH de $H$. contortus

\section{Conclusiones}

- La respuesta natural contra Haemonchus contortus permitió la selección de péptidos a través del biopanning de la biblioteca de fago que mimifican epítopos del parásito.

- Los epítopos que mimifican a DIM-1 y GAPDH confieren protección frente al parásito, a través de una respuesta IgG.

\section{Agradecimientos}

Los autores manifiestan su agradecimiento a los revisores por sus consejos para la mejora del manuscrito, a CAPES por permitir la realización de la mayor parte de los experimentos de este trabajo a través del financiamiento otorgado para la beca predoctoral de Arnielis Díaz, y a UNUBIOLAC por la beca concedida que facilitó la realización de experimentos del trabajo.

\section{Literatura Citada}

1. Ambroggio X, Jiang L, Aebig J, Obiakor H, Lukszo J, Narum D. 2013. The epitope of monoclonal antibodies blocking erythrocyte invasion by Plasmodium falciparum map to the dimerization and receptor glycan binding sites of EBA-175. PloS One 8(2): e56326. doi: 10.1371/journal.pone.0056326

2. Arnon R, Tarrab Hazdai R, Steward M. 2000. A mimotope peptide based vaccine against Schistosoma mansoni: synthesis and characterization. Immunology 101: 555-562. doi: 10.1046/ j.1365-2567.2000.00139.x

3. Assana E, Gauci CG, Kyngdon CY, Zoli AP, Dorny P, Geerts S, Lightowlers $M W$. 2010. Antibody responses to the host-protective Taenia solium oncosphere protein TSOL18 in 
pigs are directed against conformational epitopes. Parasite Immunol 32: 399-405. doi: 10.1111/j.1365-3024.2009.01197.x

4. Balic A, Cunningham C, Meeusen E. 2006. Eosinophil interactions with Haemonchus contortus larvae in the ovine gastrointestinal tract. Parasite Immunol 28: 107-115. doi: 10.1111/j.13653024.2006.00816.X

5. Bishop-Hurley SL, Strachan $K A$, Sutherland IA. 2010. The application of phage-displayed peptide libraries to ligand detection in eggs and larvae of Rhipicephalus (Boophilus) microplus. Vet Parasitol 173: 173-177. doi: 10.1016/ j.vetpar.2010.05.02

6. Bradford MM. 1976. A rapid and sensitive method for the quantitation of microgram quantities of protein utilizing the principle of protein-dye binding. Anal Biochem 72: 248-254. doi: 10.1016/00032697(76)90527-3

7. Díaz A, Arenal A, França J Gomes AL, Machado MA, Sossanovicz M, Yoshitani $U$, etr al. 2015. Optimization of an immunoenzymatic (ELISA) assay for detecting ovine antibodies against Haemonchus contortus. Rev Cubana Cienc Agric 49: 477-485.

8. Douch P, Green R, Morris C, McEwan $J$, Windon R. 1996. Phenotypic markers for selection of nematode-resistant sheep. Int J Parasitol 26: 899-911. doi: 10.1016/S0020-7519(96)80062-2

9. Erttmann KD, Kleensang $A$, Schneider E, Hammerschmidt $S$, Büttner DW, Gallin M. 2005. Cloning, characterization and DNA immunization of an Onchocerca volvulus glyceraldehyde-3-phosphate dehydrogenase (Ov-GAPDH). Biochim Biophy Acta 1741: 85-94. doi: 10.1016/j.bbadis.2004.12.010

10. Gill H, Watson D, Brandon M. 1993. Monoclonal antibody to CD4+ T cells abrogates genetic resistance to Haemonchus contortus in sheep. Immunology 78: 43-49.
11. Gnanasekar M, Rao KV, He YX, Mishra PK, Nutman TB, Kaliraj P, Ramaswamy K. 2004. Novel phage display-based subtractive screening to identify vaccine candidates of Brugia malayi. Infec Immun 72: 4707-4715. doi: 10.1128/IAI.72.8.4707-4715.2004

12. Gordon HM, Whitlock H. 1939. A new technique for counting nematode eggs in sheep faeces. J Council Sci Ind Res 12: 50-52.

13. Han $K, X u$ L, Yan $R$, Song $C$, Li X. 2012. Vaccination of goats with glyceraldehyde-3-phosphate dehydrogenase DNA vaccine induced partial protection against Haemonchus contortus. Vet Immunol Immunopathol 149: 177-185. doi: 10.1016/j.vetimm.2012.06.016

14. Knox DP, Smith W. 2001. Vaccination against gastrointestinal nematode parasites of ruminants using gut-expressed antigens. Vet Parasitol 100: 21-32. doi: 10.1016/S0304-4017(01)00480-0

15. Lacroux C, Nguyen THC, Andreoletti O, Prevot F, Grisez C, Bergeaud JP, Gruner L, et al. 2006. Haemonchus contortus (Nematoda: Trichostrongylidae) infection in lambs elicits an unequivocal Th2 immune response. Vet Res 37: 607-622. doi: 10.1051/ vetres:2006022

16. LeJambre LF, Windon RG, Smith WD. 2008. Vaccination against Haemonchus contortus: performance of native parasite gut membrane glycoproteins in Merino lambs grazing contaminated pasture. Vet Parasitol 153: 302-312. doi: 10.1016/j.vetpar.2008.01.03

17. Liu Y, Brindley PJ, Zeng Q, Li Y, Zhou J, Chen Y, Yang S, et al. 2011. Identification of phage display peptides with affinity for the tegument of Schistosoma japonicum schistosomula. Mol Biochem Parasitol 180: 86-98. doi: 10.1016/j.molbiopara.2011.09.001

18. [MAFF] Ministry of Agriculture, Fisheries and Food. 1986. Manual of veterinary parasitological laboratory 
techniques. London, UK: London: Her Majesty's Stationery Office. $160 \mathrm{p}$.

19. Melzer H, Fortugno P, Mansouri E, Felici F, Marinets A, Wiedermann G, Kollaritsch H, et al. 2002. Antigenicity and immunogenicity of phage library selected peptide mimics of the major surface proteophosphoglycan antigens of Entamoeba histolytica. Parasite Immunol 24: 321-328.

20. Muleke CI, Yan R, Sun Y, Zhao G, Xu $L, L i X .2007$. Vaccination of goats against Haemonchus contortus with a recombinant cysteine protease. Small Ruminant Res 73: 95-102. doi: 10.1016/ j.smallrumres.2006.11.006

21. Newton SE, Meeusen ENT. 2003. Progress and new technologies for developing vaccines against gastrointestinal nematode parasites of sheep. Parasite Immunol 25: 283-296. doi: 10.1046/j.1365-3024.2003.00631.x

22. Prudencio CR, Marra AO, Cardoso R, Goulart LR. 2010. Recombinant peptides as new immunogens for the control of the bovine tick, Rhipicephalus (Boophilus) microplus. Vet Parasitol 172: 122-131. doi: 10.1016/j.vetpar.2010.04.012

23. Rainbird M, Macmillan D, Meeusen EN. 1998. Eosinophil mediated killing of Haemonchus contortus larvar: effect of eosinophil activation and role of antibody, complement and interleukin 5. Parasite Immunol 20: 93-103. doi: 10.1046/j.13653024.1998.00132.x

24. Rogalski TM, Gilbert MM, Devenport D, Norman KR, Moerman DG 2003. DIM-1, a novel immunoglobulin superfamily protein in Caenorhabditis elegans, is necessary for maintaining bodywall muscle integrity. Genetics 163 : 905-915.

25. Sargison N, Jackson F, Gilleard J. 2011. Effects of age and immune suppression of sheep on fecundity, hatching and larval feeding of different strains of Haemonchus contortus. Vet J 189: 296301. doi: 10.1016/j.tvj1.2010.-08.001
26. Shakya KP, Miller JE, Lomax LG, Burnett DD. 2011. Evaluation of immune response to artificial infections of Haemonchus contortus in Gulf Coast Native compared with Suffolk lambs. Vet Parasitol 181: 239-247. doi: 10.1016/ j.vetpar.2011.03.051

27. Schallig HD, Hornok S, Cornelissen JB. 1995. Comparison of two enzyme immunoassays for the detection of Haemonchus contortus infections in sheep. Vet Parasitol 57: 329-338. doi: 10.1016/0304-4017(94)00693-7

28. Tang LF, Yi XY, Zeng XF, Wang LQ, Zhang SK. 2004. Schistosoma japonicum: isolation and identification of peptides mimicking ferritin epitopes from phage display library Acta Biochim Biophys Sin (Shanghai) 36: 206-210.

29. Tavernor AS, Smith TS, Langford CF, Graham M, Munn EA. 1992. Immune response of Clun Forest sheep to vaccination with membrane glycoproteins from Haemonchus contortus. Parasite Immunol 14: 671-675. doi: 10.1111/ j.1365-3024.1992.tb00038.x

30. Terefe G, Lacroux C, Andréoletti $O$, Grisez C, Prevot F, Bergeaud JP, Penicaud J, et al. 2007. Immune response to Haemonchus contortus infection in susceptible (INRA 401) and resistant (Barbados Black Belly) breeds of lambs. Parasite Immunol 29: 415-424. doi: 10.1111/j.1365-3024.2007.00958.x

31. Tsuji N, Kasuga-Aoki H, Isobe T, Arakawa T, Matsumoto Y. 2002. Cloning and characterisation of a highly immunoreactive $37 \mathrm{kDa}$ antigen with multi-immunoglobulin domains from the swine roundworm Ascaris suum. Int $\mathbf{J}$ Parasitol 32: 1739-1746. doi: 10.1016/ S0020-7519(02)00179-0

32. Villa-Mancera A, Quiroz-Romero H, Correa D, Ibarra F, Reyes-Pérez M, Reyes-Vivas H, López-Velázquez, $G$, et al. 2008. Induction of immunity in sheep to Fasciola hepatica with mimotopes of cathepsin L selected from a phage 
display library. Parasitology 135: 1437 1445. doi: 10.1017/S003118200800471X

33. Wang T, He G, Yang G, Fei Y, Zhang $Z$, Wang C, Yang $Z$, et al. 2008. Cloning, expression and evaluation of the efficacy of a recombinant Baylisascaris schroederi Bs-Ag3 antigen in mice. Vaccine 26: 6919-6924. doi: 10.1016/ j.vaccine.2008.09.079

34. Wardhaugh KG. 2005. Insecticidal activity of synthetic pyrethroids, organophosphates, insect growth regulators, and other livestock parasiticides: an Australian perspective. Environ Toxicol Chem 24: 789-796. doi: 10.1897/03-588.1

35. Wu HW, Hu XM, Wang Y, Kurtis JD, Zeng FJ, McGarvey ST, Wu GL, et al. 2006. Protective immunity induced by phage displayed mitochondrial related peptides of Schistosoma japonicum. Acta Trop 99: 200-207. doi: 1016/ j.actatropica.2006.08.006

36. Yan F, Xu L, Liu L, Yan R, Song X, Li $X .2010$. Immunoproteomic analysis of whole proteins from male and female adult
Haemonchus contortus. Vet J 185: 174179. doi: 10.1016/j.tvj1.2009.-05.021

37. Yan R, Sun W, Song $X, X u L, L i X$. 2013. Vaccination of goats with DNA vaccine encoding Dim-1 induced partial protection against Haemonchus contortus: a preliminary experimental study. Res Vet Sci 95: 189-199. doi: 10.1016/j.rvsc.2013.02.020

38. Yanming S, Ruofeng Y, Muleke CI, Guangwei Z, Lixin X, Xiangrui L. 2007. Vaccination of goats with recombinant galectin antigen induces partial protection against Haemonchus contortus infection. Parasite Immunol 29: 319-326. doi: 10.1111/j.13653024.2007.00949. $\mathrm{x}$

39. Zhao G, Yan R, Muleke CI, Sun Y, Xu $L, L i X .2012$. Vaccination of goats with DNA vaccines encoding H11 and IL-2 induces partial protection against Haemonchus contortus infection. Vet J 191: 94-100. doi: 10.1016/j.tvj1.2010.12.023 